\title{
Influence of Separate Confinement Heterostructure Layer on Carrier Distribution in InGaAsP Laser Diodes with Nonidentical Multiple Quantum Wells
}

\author{
Ching-Fuh LIN, Yi-Shin Su, Chao-Hsin Wu and Yu-Chia CHANG \\ Graduate Institute of Electro-Optical Engineering, Graduate Institute of Electronics Engineering and Department of Electrical Engineering, \\ National Taiwan University, Taipei, Taiwan, R.O.C.
}

(Received October 21, 2003; accepted May 27, 2004; published October 8, 2004)

The thickness of the separate confinement heterostructure ( $\mathrm{SCH}$ ) layer is found to have a significant influence on the carrier distribution among InGaAsP multiple quantum wells in laser diodes. When the SCH layer is $120 \mathrm{~nm}$ thick, the carrier distribution of the fabricated laser diodes favors quantum wells near the n-cladding layer. When the thickness of the SCH layer is reduced to $20 \mathrm{~nm}$, the carrier distribution of the fabricated laser diodes favors quantum wells near the p-cladding layer. Our experiments indicate that the carrier distribution of a fabricated laser diode can be engineered using an SCH layer of appropriate thickness. [DOI: 10.1143/JJAP.43.7032]

KEYWORDS: laser diode, separate confinement heterosturcture, carrier distribution, multiple quantum wells, nonidentical multiple quantum wells

Quantum wells are commonly used nowadays to improve the characteristics of laser diodes. However, the carrier distribution among multiple quantum wells (MQWs) is not uniform. This nonuniform carrier distribution has been theoretically predicted ${ }^{1-3)}$ and experimentally proved. ${ }^{4-7)}$ The carrier distribution among MQWs has been found to be influenced by several factors. For example, Hamp ${ }^{7,8)}$ discovered that decreasing the height or thickness of the barriers results in a more even carrier distribution among MQWs. Lin $^{9)}$ also found that the sequence of the MQW layout has a significant influence on the carrier distribution among MQWs. In the past, most investigations concluded that nonuniform carrier distributions favor QWs near the p-cladding layer. The reason was considered to be the slow mobility of holes that control the behavior of the carrier distribution. Because carrier mobility is a function of temperature, the carrier distribution depends on temperature. ${ }^{5,10)}$ However, a carrier distribution that favors QWs near the n-cladding layer has also been discovered. ${ }^{9,11)} \mathrm{A}$ new theory was thus proposed to explain such opposite behavior in the carrier distribution. ${ }^{11)}$ In this new theory, carrier transport across the separate confinement heterostructure $(\mathrm{SCH})$ layer is deemed to be an important factor that influences the carrier distribution. Here we report clear evidence of SCH-layer influence on the carrier distribution among MQWs.

Mirror-imaged nonidentical MQWs similar to those investigated in previous experiments ${ }^{11}$ ) were used here. Here, however, the thickness of the SCH layers varied. The MQW structures consist of three $60 \AA \operatorname{In}_{0.67} \mathrm{Ga}_{0.33} \mathrm{As}_{0.72} \mathrm{P}_{0.28}$ QWs and two $87 \AA \mathrm{In}_{0.53} \mathrm{Ga}_{0.47}$ As QWs that have their first quantized energies corresponding to $\lambda=1.3 \mu \mathrm{m}$ and $\lambda=$ $1.54 \mu \mathrm{m}$, respectively. The $87 \AA \mathrm{In}_{0.53} \mathrm{Ga}_{0.47} \mathrm{As} \mathrm{QWs}$ also have a second quantized state corresponding to $\lambda=1.46$ $\mu \mathrm{m}$. The QWs are separated by $150 \AA \mathrm{In}_{0.86} \mathrm{Ga}_{0.14} \mathrm{As}_{0.3} \mathrm{P}_{0.7}$ barriers. Three SCH layers of different thicknesses and two MQW layouts of opposite sequences were investigated experimentally. Therefore, there are six combinations of QW structures. Those that have two $87 \AA$ QWs located near the n-cladding layer are named A1, A2, and A3. Their QW structure is shown in Fig. 1(a). The other three QW structures that have the opposite layout sequence are named B1, B2, and B3 as shown in Fig. 1(b). A1 and B1 each have

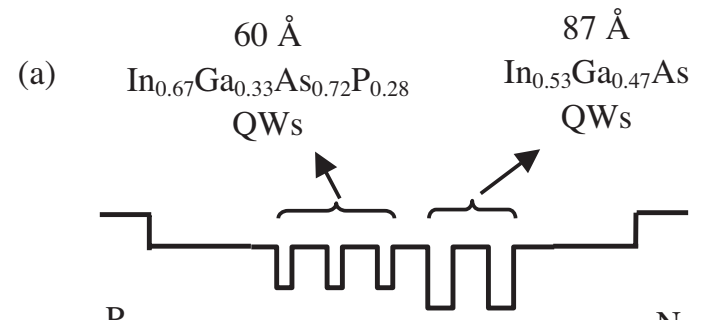

$\mathrm{P}$

$\mathrm{N}$

(b)
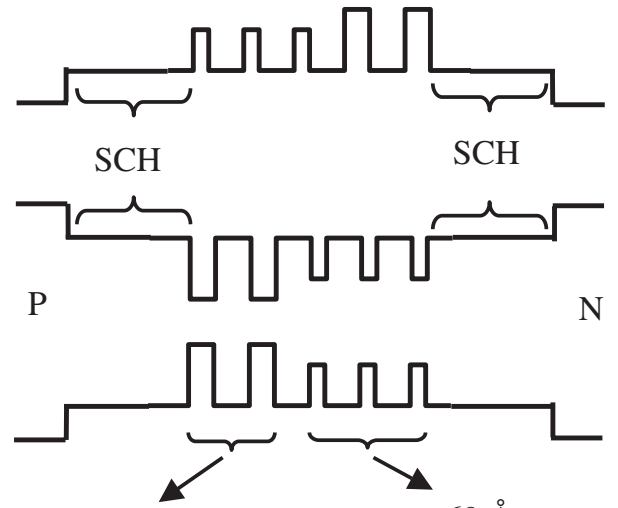

$87 \AA$

$\mathrm{In}_{0.53} \mathrm{Ga}_{0.47} \mathrm{As}$

QWs

$60 \AA$

$\mathrm{In}_{0.67} \mathrm{Ga}_{0.33} \mathrm{As}_{0.72} \mathrm{P}_{0.28}$

QWs

Fig. 1. QW structures: (a) samples A1, A2 and A3; (b) samples B1, B2 and B3.

a 120-nm-thick SCH layer. A2 and B2 each have a 60-nmthick SCH layer. A3 and B3 each have a 20-nm-thick SCH layer. These characteristics are listed in Table I. Fabry-Perot laser diodes (LDs) were fabricated on these substrates using standard processing techniques. The devices all had a $3-\mu \mathrm{m}-$

Table I. Labels of QW structures with different QW sequences and SCH thicknesses.

\begin{tabular}{lcc}
\hline $\begin{array}{c}87 \AA \text { InGaAs QWs } \\
\text { near n-cladding }\end{array}$ & $\begin{array}{c}87 \AA \text { InGaAs QWs } \\
\text { near p-cladding }\end{array}$ \\
\hline $\mathrm{SCH}=120 \mathrm{~nm}$ & A1 & B1 \\
$\mathrm{SCH}=60 \mathrm{~nm}$ & A2 & B2 \\
$\mathrm{SCH}=20 \mathrm{~nm}$ & A3 & B3 \\
\hline
\end{tabular}




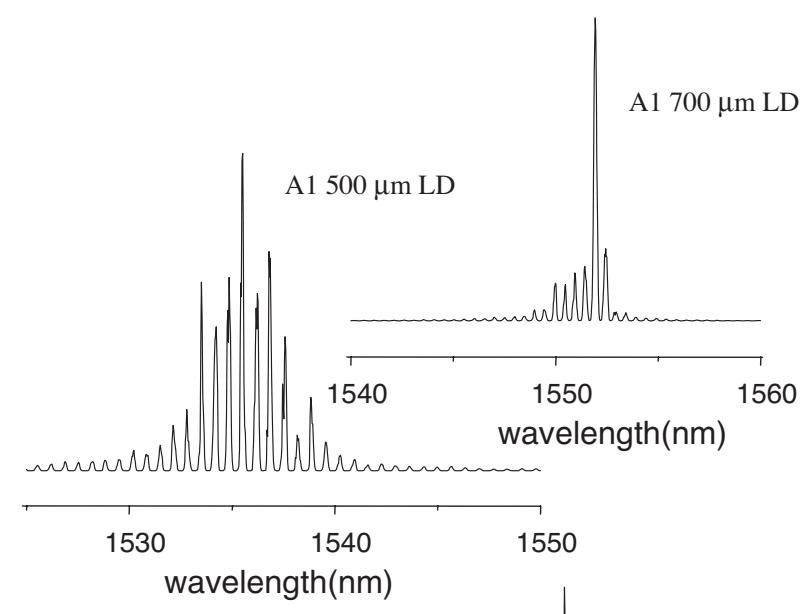

B1 $700 \mu \mathrm{m}$ LD

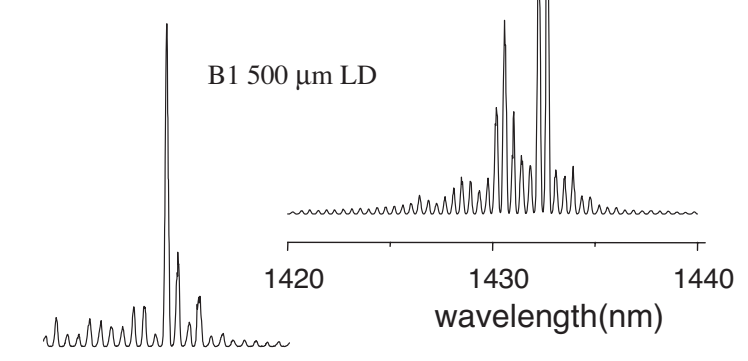

$$
\begin{array}{ll}
1380 & 1390 \\
\text { wavelength }(\mathrm{nm}) &
\end{array}
$$

Fig. 2. Lasing spectra of A1 and B1 LDs.

wide ridge waveguide. Several devices of different lengths were fabricated. No coating was applied to the device facets. LDs fabricated on substrates A1 and B1 are named A1 LDs and B1 LDs, respectively, for simple reference later. LDs fabricated on substrates A2, A3, B2, and B3 are also named in a similar manner.

A1 and B1 LDs have similar threshold currents, but their lasing wavelengths are very different due to the opposite layout of the QWs. Typical lasing spectra of A1 and B1 LDs are shown in Fig. 2. For A1 LDs, the 500- $\mu$ m-long devices and $700-\mu \mathrm{m}$-long devices have peak lasing wavelengths of approximately $1536 \mathrm{~nm}$ and $1552 \mathrm{~nm}$, respectively. The lasing wavelength of the LDs with nonidentical MQWs usually varies with device length. Having the same reflectivity of the laser facets, the short LDs have a higher threshold current density than the long LDs. ${ }^{12)}$ Because the higher threshold current density causes the gain to be blueshifted either due to the band-filling effect or due to carriers filling the QWs of high energy levels, short LDs with nonidentical MQWs oscillate at a shorter wavelength, ${ }^{8)}$ as shown by the lasing spectra of A1 LDs. Our QW structures have two types of QWs that can possibly contribute to the gain of the lasing modes. However, the lasing spectra show that only the two $87 \AA \mathrm{In}_{0.53} \mathrm{Ga}_{0.47}$ As QWs contribute to the gain of A1 LDs. In comparison with the wide separation of the first quantized energies of the two different types of QWs (corresponding to $\lambda=1300 \mathrm{~nm}$ and $\lambda=1540 \mathrm{~nm}$, respectively), the difference in peak lasing wavelength between the $500-\mu \mathrm{m}$-long and 700- $\mu \mathrm{m}$-long devices is small. Please note that the $87 \AA \mathrm{In}_{0.53} \mathrm{Ga}_{0.47}$ As QWs of the A1 QW structure are located near the n-cladding layer.

For B1 LDs, the 500- $\mu \mathrm{m}$-long devices and 700- $\mu \mathrm{m}-$ long devices have the peak lasing wavelengths of about $1383 \mathrm{~nm}$ and $1433 \mathrm{~nm}$, respectively. Again, we see that the short LDs oscillate at a shorter wavelength than the long LDs, but the difference is small compared with the wide separation of the first quantized energies of the two types of QWs. The significant shift in the peak lasing wavelengths of B1 LDs toward the short-wavelength side may be caused by two phenomena: One is the band-filling effect to the second quantized state of the $87 \AA \operatorname{In}_{0.53} \mathrm{Ga}_{0.47} \mathrm{As}$ QWs and the other is carrier injection into the $60 \AA \mathrm{In}_{0.67} \mathrm{Ga}_{0.33} \mathrm{As}_{0.72} \mathrm{P}_{0.28}$ QWs. Because the threshold currents of A1 LDs and B1 LDs are almost identical, if the band-filling effect is the only influential factor, their peak lasing wavelengths will also be almost identical. However, the experiment shows that the band-filling effect of A1 LDs causes no significant change in the peak lasing wavelegnth, but B1 LDs have a much shorter wavelength of oscillation. Thus, the carrier distribution among the two types of QWs is different for A1 and B1 LDs. That is, the QW layout sequence has a very significant influence on the carrier distribution. In B1 LDs, significant carriers are also injected into the $60 \AA \mathrm{In}_{0.67} \mathrm{Ga}_{0.33} \mathrm{As}_{0.72} \mathrm{P}_{0.28}$ QWs. Therefore, the large shift in the peak lasing wavelength in B1 LDs is caused by the gain simultaneously contributed from both the $87 \AA \mathrm{In}_{0.53} \mathrm{Ga}_{0.47} \mathrm{As}$ QWs and the $60 \AA \mathrm{In}_{0.67} \mathrm{Ga}_{0.33} \mathrm{As}_{0.72} \mathrm{P}_{0.28}$ QWs. As a result, the gain peak is located between the wavelengths corresponding to these two different types of QWs. Similar characteristics of emission spectra have also been observed for superluminescent diodes with two different types of QWs. ${ }^{9)}$ Please note that the $87 \AA \mathrm{In}_{0.53} \mathrm{Ga}_{0.47}$ As QW s of the B1 QW structure are located near the p-cladding layer.

When we compare the peak lasing wavelengths of A1 LDs and B1 LDs, the difference is significant. The peak lasing wavelength of $\mathrm{B} 1 \mathrm{LDs}$, which is much shorter than that of A1 LDs, shows that the $60 \AA \operatorname{In}_{0.67} \mathrm{Ga}_{0.33} \mathrm{As}_{0.72} \mathrm{P}_{0.28}$ QWs have more carriers in $\mathrm{B} 1 \mathrm{LD}$ s than in $\mathrm{A} 1 \mathrm{LDs}$. This indicates that the QW sequence has a significant influence on the carrier distribution. The reason is the presence of $120-\mathrm{nm}$ thick SCH layer. The thick SCH layer significantly lengthens the time it takes holes to be trapped in QWs. According to the theory proposed in ref. 11, the first type of carriers that arrive in the QW region will determine the carrier distribution. An electron-determined nonuniform carrier distribution leads to carriers favoring QWs near the n-cladding layer. For $\mathrm{A} 1 \mathrm{LDs}$, the $87 \AA \mathrm{In}_{0.53} \mathrm{Ga}_{0.47} \mathrm{As}$ QWs are near the n-cladding layer, so they contribute significantly to gain. For B1 LDs, the $87 \AA \mathrm{In}_{0.53} \mathrm{Ga}_{0.47} \mathrm{As}$ QWs are near the p-cladding layer, so their contribution to gain is reduced, leading to the peak lasing wavelength being shifted toward the transition wavelength of the $60 \AA$ $\mathrm{In}_{0.67} \mathrm{Ga}_{0.33} \mathrm{As}_{0.72} \mathrm{P}_{0.28}$ QWs.

As the thickness of the $\mathrm{SCH}$ layer is reduced, the transport time of holes across the $\mathrm{SCH}$ layer is not much longer than that of electrons. Because the capture time of holes is much shorter than that of electrons, the summation of the transport time of holes across the SCH layer and the capture time of holes is similar to the transport time and capture time of electrons. Then the carrier distribution will clearly not favor 
Table II. Threshold currents of A2 LDs and B2 LDs.

\begin{tabular}{ccc}
\hline & $\begin{array}{c}\text { Device length } \\
500 \mu \mathrm{m}\end{array}$ & $\begin{array}{c}\text { Device length }= \\
700 \mu \mathrm{m}\end{array}$ \\
\hline A2 & $42 \mathrm{~mA}$ & $37 \mathrm{~mA}$ \\
B2 & $53 \mathrm{~mA}$ & $42 \mathrm{~mA}$ \\
\hline
\end{tabular}
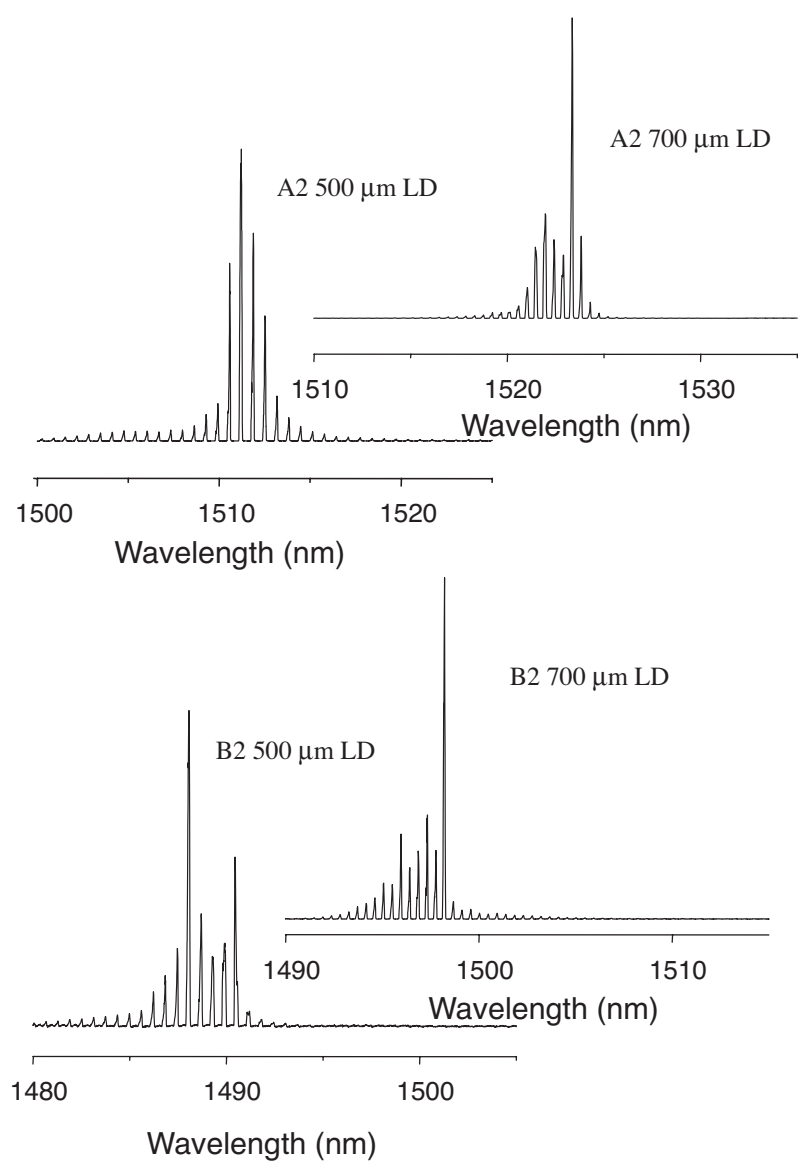

Fig. 3. Lasing spectra of A2 and B2 LDs.

QWs near the n-cladding layer. The threshold currents of A2 and B2 LDs are shown in Table II and the lasing spectra of A2 and B2 LDs are shown in Fig. 3. The threshold currents of $\mathrm{A} 2$ and $\mathrm{B} 2 \mathrm{LDs}$ of the same length do not differ much. Also, their lasing spectra are similar. For A2 LDs, the 500$\mu \mathrm{m}$-long devices and $700-\mu \mathrm{m}$-long devices have peak lasing wavelengths of approximately $1512 \mathrm{~nm}$ and $1524 \mathrm{~nm}$, respectively. For B2 LDs, the 500- $\mu \mathrm{m}$-long devices and 700$\mu \mathrm{m}$-long devices have peak lasing wavelengths of approximately $1488 \mathrm{~nm}$ and $1498 \mathrm{~nm}$, respectively. Although B2 LDs still oscillate at a shorter wavelength than A2 LDs, the difference is small, compared to the difference in peak lasing wavelength between A1 LDs and B1 LDs. The lasing spectra show that the gain for both A2 and B2 LDs is mainly contributed by the two $87 \AA \mathrm{In}_{0.53} \mathrm{Ga}_{0.47} \mathrm{As}$ QWs of these two LDs. These QWs are located near the $\mathrm{n}$-cladding layer and the p-cladding layer of A2 LDs and B2 LDs, respectively. This indicates that the preference of the carrier distribution near the $\mathrm{n}$-cladding or p-cladding layer is not obvious because the thickness of SCH layer decreases to only $60 \mathrm{~nm}$.

After careful examination, we find that the threshold current of A2 LDs is slightly less than that of B2 LDs, and that the lasing wavelength of $\mathrm{A} 2 \mathrm{LDs}$ is slightly longer than that of B2 LDs. This implies that the two $87 \AA \operatorname{In}_{0.53} \mathrm{Ga}_{0.47} \mathrm{As}$ QWs acquire more carriers for the A2 structure than for the B2 structure under the same injection current. Therefore, the carrier distribution still slightly favors QWs near the n-cladding layer for the A2 structure even though the $\mathrm{SCH}$ layer thickness is reduced to $60 \mathrm{~nm}$.

When the thickness of the SCH layer is reduced further, the preference of the carrier distribution near the n-cladding layer should be further reduced. A3 and B3 LDs each with the SCH layer thickness of $20 \mathrm{~nm}$ were fabricated. Several devices of different lengths were fabricated, but only 900$\mu \mathrm{m}$-long devices showed lasing behavior. Because of the narrow SCH layer, the lasing mode is extended to the doped region, which increases the degree of optical absorption and thus the threshold current density. In addition, the thin SCH layer may cause the dopants in the cladding layer to diffuse into the QWs, leading to the decrease in gain. Thus, short LDs cannot oscillate easily. The lasing spectra of $900-\mu \mathrm{m}-$ long LDs are shown in Fig. 4. Their peak lasing wavelengths are approximately $1523 \mathrm{~nm}$ and $1521 \mathrm{~nm}$ for A3 LDs and B3 LDs, respectively. These lasing spectra are similar to those of A2 and B2 LDs of $900 \mu \mathrm{m}$ device length. Typical $L-I$ curves of A2, B2, A3, and B3 LDs of $900 \mu \mathrm{m}$ device length are shown in Fig. 5. The threshold currents of A3 and B3 LDs are approximately $40-50 \mathrm{~mA}$, slightly larger than those of A2 and B2 LDs. The reasons for this are discussed above. The lasing spectra show that gain is mainly contributed by the $87 \AA \operatorname{In}_{0.53} \mathrm{Ga}_{0.47} \mathrm{As}$ QWs irrespective of whether they are located near the $\mathrm{n}$-cladding layer or the p-cladding layer.
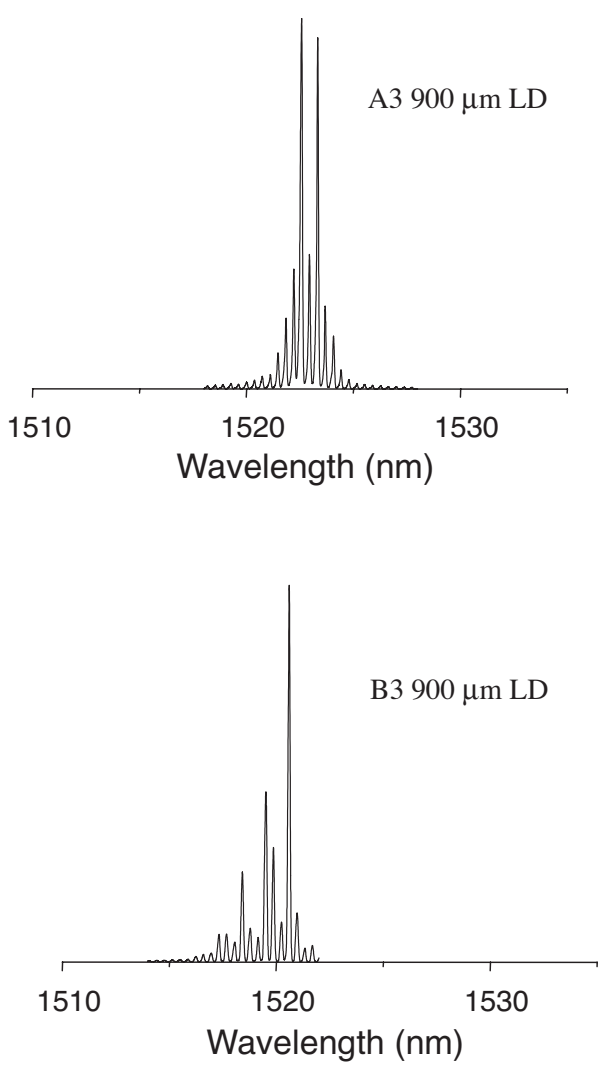

Fig. 4. Lasing spectra of $\mathrm{A} 3$ and $\mathrm{B} 3 \mathrm{LDs}$. 

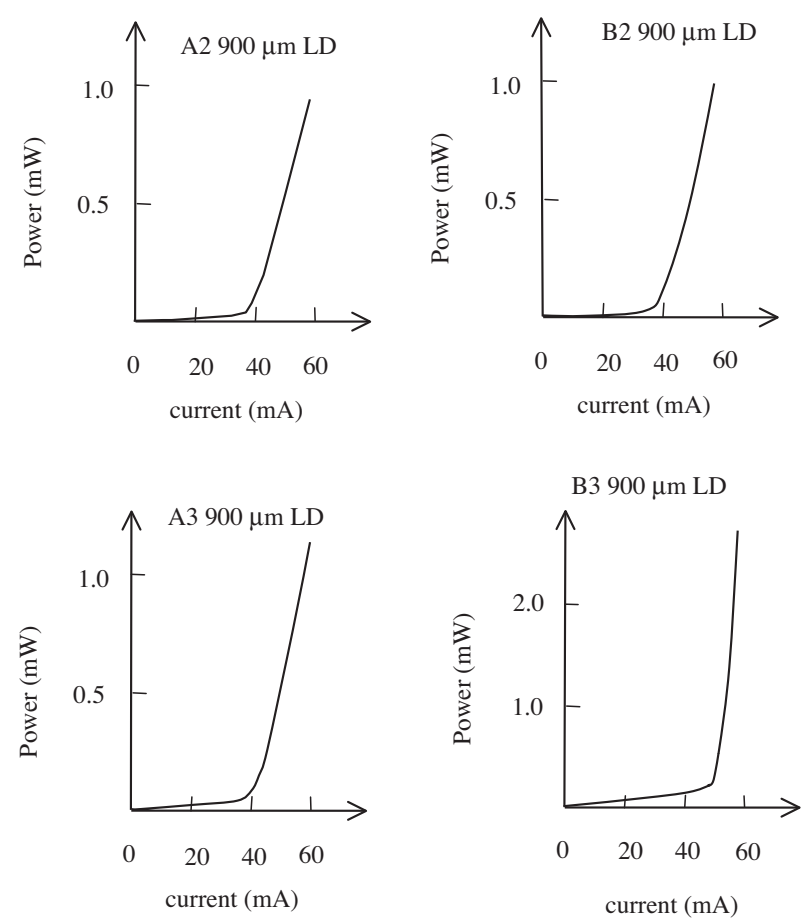

Fig. 5. $L-I$ curves of A2, B2, A3 and B3 LDs.

Table III. Slope efficiencies of A2, B2, A3 and B3 LDs.

\begin{tabular}{cc}
\hline & Slope efficiency \\
\hline A2 & $0.053 \mathrm{~W} / \mathrm{A}$ \\
B2 & $0.056 \mathrm{~W} / \mathrm{A}$ \\
A3 & $0.063 \mathrm{~W} / \mathrm{A}$ \\
B3 & $0.31 \mathrm{~W} / \mathrm{A}$ \\
\hline
\end{tabular}

Thus, unlike the A1 and B1 LDs, the carrier distribution does not favor for QWs near the n-cladding layer as the $\mathrm{SCH}$ layer is only $20 \mathrm{~nm}$ thick.

The slope efficiency of those LDs is shown in Table III. In the calculation of the slope efficiency, only the light emitted from one facet is taken into account. For A2, B2 and A3 LDs, the slope efficiency is less than $0.1 \mathrm{~W} / \mathrm{A}$ per facet. In comparison, the slope efficiency of B3 LDs is as high as $0.31 \mathrm{~W} / \mathrm{A}$ per facet. The high slope efficiency of B3 LDs compared with that of A2, B2 and A3 LDs can be explained by the following reasons. First, B3 LDs have a thin $\mathrm{SCH}$ layer, which causes holes to reach the $\mathrm{QW}$ region in a very short time. Secondly, the capture time of holes is much shorter than that of electrons. Therefore, holes occupy the QWs before electrons do, leading to a carrier distribution which is determined by holes and favors QWs near the p-cladding layer. Thirdly, carriers tend to fill up low-energy levels first. Because B3 LDs have the $87 \AA \mathrm{In}_{0.53} \mathrm{Ga}_{0.47} \mathrm{As}$ QWs located near the p-cladding layer, the above factors all favor carriers filling these QWs. Therefore, B3 LDs have more injected carriers contributing to the gain at the wavelength corresponding to the $87 \AA \mathrm{In}_{0.53} \mathrm{Ga}_{0.47}$ As QWs, and thus the efficiency is increased. The thin $\mathrm{SCH}$ layer of the B3 structure can increase the optical absorption of the laser mode and thus threshold current. However, the fast process of stimulated emission at long wavelengths quickly consumes carriers injected into the corresponding QWs. Then the above factors favoring the $87 \AA \operatorname{In}_{0.53} \mathrm{Ga}_{0.47}$ As QWs of B3 LDs cause the further injection of carriers to mostly fill these QWs. As a result, the preference of carriers to fill the $87 \AA \mathrm{In}_{0.53} \mathrm{Ga}_{0.47} \mathrm{As} \mathrm{QW}$ of B3 LDs is even more prominent when the LD is lasing and this effect overrides the increased in the degree of optical absorption. In comparison, the A3 LDs do not behave in the same way because their $87 \AA$ $\mathrm{In}_{0.53} \mathrm{Ga}_{0.47} \mathrm{As} \mathrm{QWs}$ are located near the n-cladding layer.

From the above experiments, we know that a thick SCH layer causes the carrier distribution to favor QWs near the n-cladding layer, while a thin SCH layer causes the carrier distribution to favor QWs near the p-cladding layer. Therefore, with a proper thickness of SCH layer, a uniform carrier distribution among the MQWs can be obtained.

In conclusion, the thickness of a $\mathrm{SCH}$ layer is found to have a significant influence on the carrier distribution among InGaAsP MQWs. For a SCH layer of $120 \mathrm{~nm}$ thickness, the layout sequence of nonidentical MQWs has a strong influence on the obtained lasing spectra. The measured spectra show that the carrier distribution favors QWs near the n-cladding layer. When the SCH layer thickness is reduced to $60 \mathrm{~nm}$ or less, the layout sequence of nonidentical MQWs has a decreasing influence on the obtained lasing spectra. As the SCH layer thickness is reduced to only $20 \mathrm{~nm}$, the fabricated LDs show that the carrier distribution favors QWs near the p-cladding layer. Therefore, the carrier distribution among the MQWs can be engineered using an $\mathrm{SCH}$ layer of appropriate thickness.

This work is supported in part by the National Science Council, Taipei, Taiwan, R.O.C. under contract No. 922125-E-002-012.

1) R. Nagarajan, T. Fukushima, S. W. Corzine and J. E. Bowers: Appl. Phys. Lett. 59 (1991) 1835.

2) A. Hangleiter, A. Grabmaier and G. Fuchs: Appl. Phys. Lett. 62 (1993) 2316.

3) N. Tessler and G. Eisenstein: IEEE J. Quantum Electron. 29 (1993) 1586.

4) K. Frojdh, S. Marcinkevicius, U. Olin, C. Silfvenius, B. Stalnacke and G. Landgren: Appl. Phys. Lett. 69 (1996) 3695.

5) H. Yamazaki, A. Tomita and M. Yamaguchi: Appl. Phys. Lett. 71 (1997) 767.

6) B.-L. Lee, C.-F. Lin, J.-W. Lai and W. Lin: Electron. Lett. 34 (1998) 1230.

7) M. J. Hamp, D. T. Cassidy, B. J. Robinson, Q. C. Zhao, D. A. Thompson and M. Davies: IEEE Photon. Technol. Lett. 10 (1998) 1380.

8) M. J. Hamp, D. T. Cassidy, B. J. Robinson, Q. C. Zhao and D. A. Thompson: IEEE Photon. Technol. Lett. 12 (2000) 134.

9) C.-F. Lin, B.-R. Wu, L.-W. Laih and T.-T. Shih: Opt. Lett. 26 (2001) 1099.

10) P. M. Smowton, G. M. Lewis, A. Sobiesierski, P. Blood, J. Lutti and S. Osbourne: Appl. Phys. Lett. 83 (2003) 419.

11) C.-F. Lin, B.-R. Wu, L.-W. Laih and T.-T. Shih: Jpn. J. Appl. Phys. 42 (2003) 5557.

12) S. L. Chuang: Physics of Optoelectronic Devices (John Wiley \& Sons, New York, 1995) Chap. 10. 